\title{
Energy dependence of $\phi$-meson production and elliptic flow in Au+Au collisions at STAR
}

\author{
Md. Nasim (for the STAR collaboration)* \\ School of Physical Science \\ National Institute of Science Education and Research \\ Bhubaneswar-751 005, India \\ E-mail: nasimercf.rhic.bnl.gov
}

\begin{abstract}
We present, the measurements of the $\phi$-meson production and elliptic flow $\left(v_{2}\right)$ at mid-rapidity in $\mathrm{Au}+\mathrm{Au}$ collisions at $\sqrt{s_{N N}}=7.7-200 \mathrm{GeV}$ using the STAR detector in the years 2010 and 2011. The energy dependence of nuclear modification factors $\left(R_{\mathrm{CP}}\right)$ is presented. At the intermediate transverse momentum $\left(p_{T}\right)$ the $\Omega / \phi$ ratio shows a different trend for $\sqrt{s_{N N}}=11.5 \mathrm{GeV}$ compared to higher beam energies. This may suggest change of particle production mechanism at $\sqrt{s_{N N}}$ $=11.5 \mathrm{GeV}$. The number-of-constituent quark (NCQ) scaling of $v_{2}$ has been studied at various beam energies. The NCQ scaling holds for all identified particles for $\sqrt{s_{N N}} \geq 19.6 \mathrm{GeV}$, which can be considered as an evidence of partonic collectivity. We observe at $\sqrt{s_{N N}}=7.7$ and 11.5 $\mathrm{GeV}$, the $\phi$-meson $v_{2}$ falls off the trend from the other hadrons at highest measured $p_{T}$ values by $1.8 \sigma$ and $2.3 \sigma$, respectively. This may indicate that the hadronic interaction plays an important role at lower beam energies.
\end{abstract}

8th International Workshop on Critical Point and Onset of Deconfinement,

March 11 to 15, 2013

Napa, California, USA

* Speaker. 


\section{Introduction}

The $\phi$ vector meson is the lightest bound state of $s$ and $\bar{s}$ quarks. The interaction cross-section of the $\phi$ meson with non-strange hadrons is expected to have a small value [1] and therefore its production should be less affected by the later stage hadronic interactions in the evolution of the system formed in heavy-ion collisions. The $\phi$ meson seems to freeze out early compared to other light hadrons ( $\pi, K$ and $p$ ) [1]. The life time of the $\phi$ meson is $\sim 42 \mathrm{fm} / c$. Because of longer life time the $\phi$ meson will mostly decay outside the fireball and therefore its daughters will not have much time to re-scatter in the hadronic phase. The elliptic flow $\left(v_{2}\right)$, a measure of the anisotropy in momentum space, for $\phi$ meson can be used to probe the dynamics of the early stage of heavyion collisions [2]. For the $\phi$-meson $v_{2}$, effect of later stage hadronic interaction is small [3, 4]. Therefore, the $\phi$ meson can be considered as a clean probe to study the QCD phase diagram in the Beam Energy Scan (BES) program at the Relativistic Heavy Ion Collider (RHIC) [5].

\section{Data sets and methods}

The results presented here are based on data collected at $\sqrt{s_{N N}}=7.7,11.5,19.6,27,39,62.4$ and $200 \mathrm{GeV}$ in $\mathrm{Au}+\mathrm{Au}$ collisions by the STAR detector for a minimum bias trigger in the years of 2010 and 2011. The Time Projection Chamber (TPC) and Time of Flight (TOF) detectors with full $2 \pi$ coverage were used for particle identification in the central pseudo-rapidity $(\eta)$ region $(|\eta|<1.0)$. $\phi$ mesons are identified using the invariant mass technique from their decay to $K^{+}+$ $K^{-}$(branching ratio is $49.04 \pm 0.6 \%$ ). Mixed event technique has been used for combinatorial background estimation [6]. The $\eta$-sub event plane method [7] using TPC tracks has been applied to measure the elliptic flow. In this method, one defines the event flow vector for each particle based on particles measured in the opposite hemisphere in pseudo-rapidity $(\eta)$. An $\eta$ gap of $|\eta|<0.05$ between positive and negative pseudo-rapidity sub-events has been introduced to suppress non-flow effects.

\section{Results}

The $R_{\mathrm{CP}}(0-10 \% / 40-60 \%)$ of $\phi$ mesons at mid-rapidity $(|y|<0.5)$ in $\mathrm{Au}+\mathrm{Au}$ collisions at $\sqrt{s_{N N}}$ $=7.7-39 \mathrm{GeV}$ are presented in the panel (a) of Fig. 1 . The $R_{\mathrm{CP}}(0-05 \% / 40-60 \%)$ at $\sqrt{s_{N N}}=200$ $\mathrm{GeV}$ are taken from previous STAR measurements [8]. The $R_{\mathrm{CP}}$ is defined as the ratio of the particles yield in the central to peripheral collisions normalized by number of binary collisions $\left(N_{\text {bin }}\right)$. The value of $N_{\text {bin }}$ is calculated from the Monte Carlo Glauber simulation [9]. If $R_{\mathrm{CP}}$ is equal to one, then the nucleus nucleus collision is simply superposition of nucleon nucleon collisions. Deviation of $R_{\mathrm{CP}}$ from the unity would imply contribution from the nuclear medium effects. Because of the energy loss of the partons traversing the high density QCD medium the $R_{\mathrm{CP}}$ of $\phi$ mesons goes below unity at $200 \mathrm{GeV}$ [1]. From the Fig. 1 one can see at the intermediate $p_{T}, R_{\mathrm{CP}}$ goes above unity with decrease in beam energy. This indicates that at lower beam energy the parton energy loss effect could be less important.

The panel (b) of Fig. 1 shows the baryon-to-meson ratio, $\left(\Omega^{-}+\bar{\Omega}^{+}\right) / 2 \phi$, as a function of $p_{T}$ in $\mathrm{Au}+\mathrm{Au}$ collisions at $\sqrt{s_{N N}}=11.5 \mathrm{GeV}$ to $200 \mathrm{GeV}$. The data points for $200 \mathrm{GeV}$ are taken from 

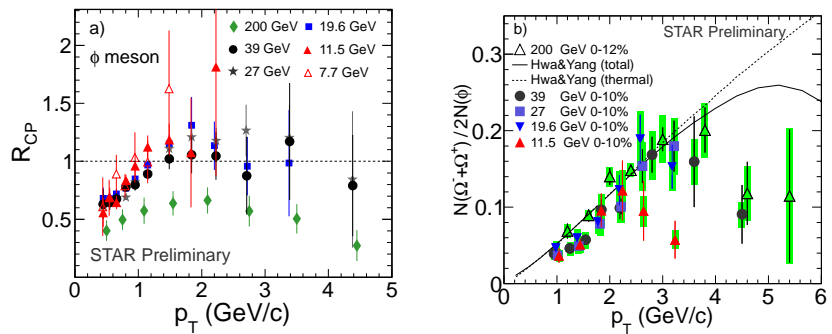

Figure 1: (Color online) Panel (a): The $R_{\mathrm{CP}}$ as function of $p_{T}$ at mid-rapidity $(|y|<0.5)$ in the $\mathrm{Au}+\mathrm{Au}$ collision at various beam energy. Error bars are only statistical uncertainties. Panel (b): The baryon-tomeson ratio, $\left(\Omega^{-}+\bar{\Omega}^{+}\right) / 2 \phi$, as a function of $p_{T}$ at mid-rapidity $(|y|<0.5)$. Green bands are the systematic errors and vertical lines are statistical errors.

Ref. [8]. The dashed lines are the results from the recombination model calculations with thermal strange quarks [10]. In Au+Au central collisions at $\sqrt{{S_{N N}}}=200 \mathrm{GeV}$, the ratios of $\left(\Omega^{-}+\bar{\Omega}^{+}\right) / 2 \phi$ in the intermediate $p_{T}$ range are explained by the recombination model with thermal strange quarks. The ratios $\left(\Omega^{-}+\bar{\Omega}^{+}\right) / 2 \phi$ for $\sqrt{s_{N N}} \geq 19.6 \mathrm{GeV}$ show similar trend. But at $\sqrt{s_{N N}}=11.5 \mathrm{GeV}$, the ratio at the highest measured $p_{T}$ shows a deviation from the trend of other energies. This may suggest a change in $\Omega$ and/or $\phi$ production mechanism at $\sqrt{s_{N N}}=11.5 \mathrm{GeV}$.

It has been observed from RHIC measurements that when $v_{2}$ and corresponding $p_{T}$ are scaled by number of constituent quarks of the hadrons, the measured $v_{2}$ values at the intermediate $p_{T}$ are consistent with expectations from parton coalescence or recombination models $[14,15]$. This is known as NCQ scaling and considered as a signature of partonic collectivity. Figure 2 shows $v_{2}$

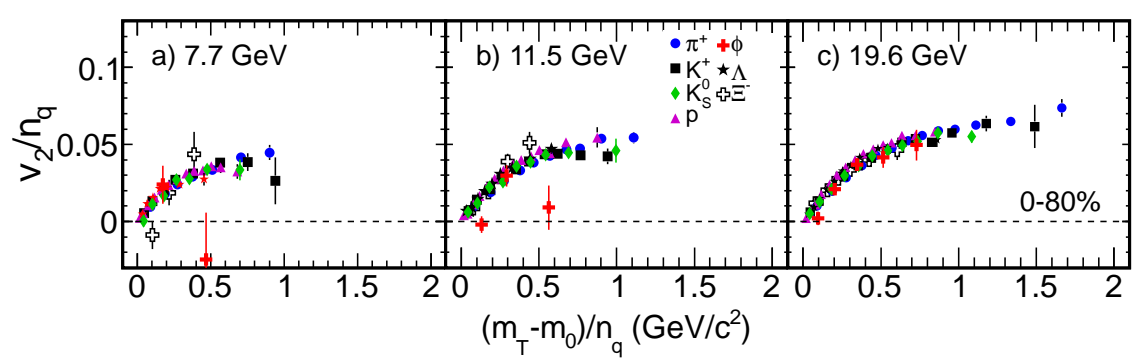

Figure 2: (Color online) The elliptic flow scaled by number-of-constituent quark $\left(n_{q}\right)$ as a function of $\left(m_{T}-m_{0}\right) / n_{q}$ for selected particles in the Au+Au collision at $\sqrt{s_{N N}}=7.7,11.5$ and $19.6 \mathrm{GeV}$ for $0-80 \%$ centrality $[12,13]$. Error bars are only statistical uncertainties.

divided by number of constituent quark as function of $\left(m_{T}-m_{0}\right) / n_{q}$, where $m_{T}=\sqrt{\left(p_{T}^{2}+m_{0}^{2}\right)}$ is the transverse mass and $m_{0}$ is the mass of the hadron, at $\sqrt{s_{N N}}=7.7,11.5$ and $19.6 \mathrm{GeV}$. The NCQ scaling holds fairly well at $\sqrt{s_{N N}} \geq 19.6 \mathrm{GeV}$ (results for $\sqrt{s_{N N}}>19.6 \mathrm{GeV}$ are not shown here). This could be considered as a signature of partonic collectivity. However, we observe at $\sqrt{s_{N N}}=7.7$ and $11.5 \mathrm{GeV}$ that the $\phi$-meson $v_{2}$ deviates from the trend of the other hadrons at highest measured 
$p_{T}$ values by $1.8 \sigma$ and $2.3 \sigma$, respectively. Due to the small hadronic interaction cross-section, $v_{2}$ of $\phi$ meson mostly reflect collectivity from the partonic phase $[3,4]$. So the small magnitude of the $\phi$-meson $v_{2}$ at $\sqrt{s_{N N}} \leq 11.5 \mathrm{GeV}$ could be the effect for a system, where hadronic interactions are more important. But more statistics are needed at $\sqrt{s_{N N}}=7.7$ and $11.5 \mathrm{GeV}$ for $\phi$-meson $v_{2}$ measurement to draw a clear conclusion and therefore $\phi$ measurement would be one of the focuses in the proposed BES phase II program.

\section{Summary}

We report the study of $\phi$-meson production and elliptic flow at mid-rapidity in $\mathrm{Au}+\mathrm{Au}$ collisions at $\sqrt{s_{N N}}=7.7-200 \mathrm{GeV}$ recorded by the STAR detector. At the intermediate $p_{T}$, the nuclear modification factor $R_{\mathrm{CP}}$ of $\phi$ increases with decreasing beam energies, indicating that the partonic energy loss effect becomes less important at lower beam energies. The ratios of $\left(\Omega^{-}+\bar{\Omega}^{+}\right) / 2 \phi$ in the intermediate $p_{T}$ range show a different trend at $11.5 \mathrm{GeV}$ compared to those for the higher beam energies. This may suggest a change of particle production mechanism at lower beam energy. The NCQ scaling holds for $\sqrt{s_{N N}} \geq 19.6 \mathrm{GeV}$. We observe at $\sqrt{s_{N N}}=7.7$ and $11.5 \mathrm{GeV}$ the $\phi$-meson $v_{2}$ show deviation from the other hadrons at highest measured $p_{T}$ values by $1.8 \sigma$ and $2.3 \sigma$, respectively. This may indicate that the contribution to the collectivity from partonic phases decreases at lower beam energies.

\section{Acknowledgements}

Financial support from DST SwarnaJayanti project, Government of India is gratefully acknowledged.

\section{References}

[1] STAR Collaboration, J. Adams et al., Nuclear Physics A, 757102 (2005).

[2] P.F. Kolb et al. Nucl. Phys. A715, 653c (2003).

[3] B. Mohanty and N. Xu , J. Phys. G 36, 064022 (2009).

[4] Md. Nasim, B. Mohanty and N. Xu, Phys. Rev. C 87, 014903 (2013).

[5] STAR Collaboration, M.M.Aggarwal et al., arXiv:1007.2613 [nucl-ex].

[6] STAR Collaboration, B. I. Abelev et al., Phys. Lett. B 673, 183 (2009).

[7] A. M. Poskanzer and S. A. Voloshin, Phys. Rev. C 58, 1671 (1998).

[8] STAR Collaboration, B. I. Abelev et al., Phys. Rev. C 49, 064903 (2009).

[9] STAR Collaboration, B. I. Abelev et al., Phys. Rev. C79, 034909 (2009).

[10] R.C. Hwa and C.B. Yang, Phys. Rev. C 75, 054904 (2007).

[11] T. Hirano et al., Phys. Rev. C 77, 044909 (2008).

[12] STAR Collaboration, L. Adamczyk et al., arXiv:1301.2348 [nucl-ex].

[13] STAR Collaboration, L. Adamczyk et al., Phys. Rev. Lett. 110, 142301 (2013).

[14] D. Molnar and S. A. Voloshin, Phys. Rev. Lett. 91, 092301 (2003).

[15] STAR Collaboration, J. Adams et al., Phys. Rev. Lett. 92, 052302 (2004). 